\title{
Proteoglycan production is required in initial stages of new cartilage matrix formation but inhibits integrative cartilage repair
}

\begin{tabular}{|c|c|}
\hline Journal: & Journal of Tissue Engineering and Regenerative Medicine \\
\hline Manuscript ID: & TERM-08-0101.R1 \\
\hline Wiley - Manuscript type: & Research Article \\
\hline $\begin{array}{r}\text { Date Submitted by the } \\
\text { Author: }\end{array}$ & 10-Dec-2008 \\
\hline Complete List of Authors: & $\begin{array}{l}\text { Bastiaansen-Jenniskens, Yvonne; Erasmus MC University Medical } \\
\text { Center, Orthopaedics; TNO Quality of Life, Business Unit } \\
\text { BioSciences } \\
\text { Koevoet, Wendy; Erasmus MC, University Medical Centre, } \\
\text { Otorhinolaryngology } \\
\text { Feijt, Carola; Erasmus MC University Medical Center, Orthopaedics } \\
\text { Bos, Pieter; Erasmus MC University Medical Center, Orthopaedics } \\
\text { Verhaar, Jan; Erasmus MC University Medical Center, Orthopaedics } \\
\text { van Osch, Gerjo; Erasmus MC University Medical Center, } \\
\text { Orthopaedics; Erasmus MC, University Medical Center, } \\
\text { Otorhinolaryngology } \\
\text { DeGroot, Jeroen; TNO Quality of Life, Business Unit BioSciences }\end{array}$ \\
\hline Keywords: & $\begin{array}{l}\text { glycosaminoglycan, collagen, collagen cross-links, cartilage matrix, } \\
\text { chondrocyte, integrative repair }\end{array}$ \\
\hline
\end{tabular}

\section{s scholarONE" Manuscript Central}




\title{
Proteoglycan production is required in initial stages of new cartilage matrix
} formation but inhibits integrative cartilage repair

\author{
Y.M. Bastiaansen-Jenniskens ${ }^{1,2}$; W. Koevoet ${ }^{3}$; C. Feijt ${ }^{1}$; P.K. Bos ${ }^{1}$; J.A.N. Verhaar ${ }^{1}$; \\ G.J.V.M. VanOsch ${ }^{1,3} ;$ J. DeGroot ${ }^{2}$
}

1: Erasmus MC, University Medical Centre Rotterdam, dept. of Orthopaedics, the Netherlands

2: TNO Quality of Life, Business Unit BioSciences, Leiden, the Netherlands

3: Erasmus MC, University Medical Centre Rotterdam, dept. of Otorhinolaryngology, the Netherlands

Keywords: glycosaminoglycan, collagen, collagen cross-links, cartilage matrix, chondrocyte, integrative repair

Supported by a grant of The Dutch Arthritis Association (Reumafonds), NR 02-2-40 


\begin{abstract}
The optimal stimulus to repair or regenerate cartilage is not known. We therefore modulated collagen deposition, collagen cross-linking and GAG deposition simultaneously during cartilage matrix production and integrative repair, creating more insight in their role in cartilage repair processes.

Insuline-like Growth Factor 1 (IGF-1, increases proteoglycan and collagen synthesis), Beta-aminopropionitrile (BAPN, a reversible inhibitor of collagen crosslinking) and para-nitrophenyl-beta-d-xyloside (PNPX, interferes with proteoglycan production) were used. Bovine articular chondrocytes were cultured in alginate beads for three weeks with or without IGF-1, BAPN or PNPX alone and in all possible combinations followed by three weeks in control medium. DNA content, GAG and collagen deposition and collagen cross-links were determined. Cartilage constructs were cultured under same conditions and histologically analysed for integration of two opposing cartilage matrices.
\end{abstract}

In alginate cultures, inhibition of collagen cross-linking with BAPN in combination with promotion of matrix synthesis using IGF1 was most beneficial for matrix deposition. Addition of PNPX was always detrimental for matrix deposition. For integration of opposing cartilage constructs, the combination of BAPN, IGF1 and temporary prevention of proteoglycan formation with PNPX was most beneficial.

When a new matrix is produced, proteoglycans are important to retain collagen in the matrix. When two already formed cartilage matrices have to integrate, temporary absence of proteoglycans and temporary inhibition of collagen cross-linking might be more beneficial in combination with stimulation of collagen production by for example IGF1. Therefore, the choice of soluble factors to promote cartilage regeneration depends on the type of therapy that will be used. 


\section{Introduction}

Once damaged, adult articular cartilage has a poor repair capacity, which is probably due to the ineffective repair of the collagen network, since proteoglycan depletion is often reversible (Lafeber 1992). Although collagen turnover is increased in osteoarthritis $(\mathrm{OA})$, this does not lead to the formation of a functional network (Aigner 1993; Billinghurst 1997; Nelson 1998; Verzijl 2000). This suggests an activated repair mechanism in OA that appears however ineffective in repairing or maintaining the ECM homeostasis.

Several strategies are under investigation to promote matrix regeneration in cartilage repair. Growth factors are used to modulate matrix production, by directly adding them to chondrocytes in culture (Gooch 2002; Veilleux 2005) or by inducing over expression of the growth factor of interest (Madry 2002; Kaul 2006). For example, we and others found that addition of IGF1 to chondrocytes in culture resulted in more proteoglycans and collagen than in the control condition without IGF1 (Blunk 2002; Mauck 2003; De Mattei 2004; Jenniskens 2006)

In addition to growth factors, modulation of collagen network formation is also employed to understand cartilage matrix formation and functionality, for example by the inhibition of collagen cross-linking by inhibition of lysyl oxidase (LOX) with $\beta$ aminopropionitrile (BAPN). It was found that collagen cross-links are important for the integrative repair and adhesive strength of cartilage (DiMicco 2002) and that transient inhibition of the formation of these cross-links improved integrative repair and collagen cross-link maturation (McGowan 2005). In cultures of chondrocytes in alginate, inhibition of collagen cross-link formation with BAPN resulted in an increase of collagen production (Beekman 1997; Wong 2002; Bastiaansen-Jenniskens 2008a). In concordance with earlier explant studies (McGowan 2005), transient 
inhibition of LOX in alginate cultures resulted in accelerated cross-link maturation and improved functionality of the newly formed matrix (Bastiaansen-Jenniskens 2008a). The influence of glycosaminoglycans (GAGs) on cartilage growth and matrix production is also under investigation, but gets less attention than collagen. GAG depletion in cartilage explants resulted in less expansive growth and a more mature matrix with increased tensile integrity (Asanbaeva 2007). Preventing GAGs from binding to the proteoglycan core protein with para-nitrophenyl xyloside (PNPX) in the newly forming cartilage matrix of chondrocytes cultured in alginate resulted in less collagen deposition and a decrease in stiffness and ability to hold water (Bastiaansen-Jenniskens 2008b). The difference between these two studies is that in the explant study collagen was already deposited and cross-linked, whereas in the alginate cultures no collagen was yet deposited when GAG incorporation was inhibited. Both studies however indicate that modulating GAGs can influence the collagen network in an already existing cartilage matrix or during new cartilage matrix synthesis.

Most of the approaches mentioned above focused on modulating one matrix component (either collagen or proteoglycans). However, increasing collagen synthesis alone is not sufficient for cartilage repair. Because of the differential and potentially complementary effects of these approaches, our hypothesis was that combining IGF1, BAPN and PNPX in one experiment has the potential to stimulate matrix production and integrative repair more than each single component. Chondrocytes cultured in alginate were used to examine the effect of the soluble factors on new matrix production, either alone or in all possible combinations. Cartilage explants were used to examine the effect of the factors mentioned above on integration of two existing cartilage matrices. 


\section{Materials and Methods}

\subsection{Cell culture}

Articular cartilage was harvested from the metacarpophalangeal joints of calves aged 6-12 months. Chondrocytes were isolated, suspended in alginate in a concentration of $4 \times 10^{6}$ cells per $\mathrm{mL}$ of alginate, and alginate beads were made as described previously (Jenniskens 2006). Beads were cultured in a six-well plate (BD Falcon, Bedford, MA, USA), with $75 \mu \mathrm{l} /$ bead DMEM/F12 supplemented with $10 \%$ fetal bovine serum (GibcoBRL), $50 \mu \mathrm{g} / \mathrm{ml}$ L-asorbic acid 2-phosphate (Sigma), $50 \mu \mathrm{g} / \mathrm{ml}$ gentamicin and $1.5 \mu \mathrm{g} / \mathrm{ml}$ fungizone (both GibcoBRL). Chondrocytes were cultured for 21 days in the presence of $25 \mathrm{ng} / \mathrm{ml}$ IGF-1, $0.25 \mathrm{mM}$ BAPN and/or $0.25 \mathrm{mM}$ PNPX based on previous results (Jenniskens 2006; Bastiaansen-Jenniskens 2008a; Bastiaansen-Jenniskens 2008b) followed by 21 days in control medium. As known from these previous studies, BAPN inhibits collagen cross-linking and PNPX prevents incorporation of GAGs into the matrix. In the 21 days of additional culture without supplements, cross-links had the ability to form and GAGs to incorporate into the matrix. Culture medium was changed three times a week. Alginate beads were harvested after 42 days of culture.

\subsection{Preparation of cartilage explants for integration study}

Articular cartilage samples were harvested from the metacarpophalangeal joints of calves aged 6-12 months. Full-thickness cartilage explants of $8 \mathrm{~mm}$ diameter and with a thickness of $0.9-1.2 \mathrm{~mm}$ were prepared using a dermal biopsy punch and scalpel. From the centre of the explants, 3-mm cores were punched out. All samples (both outer ring and inner core) were incubated for 24 hours in $10 \mathrm{U} / \mathrm{ml}$ highly purified collagenase VII (Sigma-Aldrich Chemie BV, Zwijndrecht, The Netherlands) in DMEM- 
F12 with $10 \%$ fetal calf serum based on previous results (Bos 2002; van de Breevaart Bravenboer 2004; Janssen 2006). From the study of Bos et al (Bos 2002), it was concluded that there is some loss of matrix proteins by digesting for 24 hours with highly purified collagenase, but only on the locations where proteoglycans are lost, i.e. the area of the wound edge. Pre-treatment with collagenase is beneficial for integration because it increases the number of viable cells at the wound edge thereby enabling integration of the wound edges. All the cartilage explants were pretreated with the collagenase irrespective of the condition. After incubation, the samples were washed three times for $10 \mathrm{~min}$ in culture medium, and the 3-mm inner cores were reimplanted in their accompanying 8-mm outer rings. Constructs were cultured in parallel to the alginate beads for 21 days in the presence of $25 \mathrm{ng} / \mathrm{ml}$ IGF1, $0.25 \mathrm{mM}$ BAPN and/or $0.25 \mathrm{mM}$ PNPX followed by 21 days in control medium. Constructs were cultured in $1.5 \mathrm{ml}$ DMEM/F12 per construct supplemented with $10 \%$ fetal bovine serum (GibcoBRL), $50 \mu \mathrm{g} / \mathrm{ml}$ L-asorbic acid 2-phosphate (Sigma), 50 $\mu \mathrm{g} / \mathrm{ml}$ gentamicin and $1.5 \mu \mathrm{g} / \mathrm{ml}$ fungizone (both GibcoBRL) in the presence of 25 ng/ml IGF-1, $0.25 \mathrm{mM}$ BAPN and/or $0.25 \mathrm{mM}$ PNPX during the first 21 days. After 42 days, constructs were harvested an immediately fixed in $4 \%$ phosphate buffered formalin.

\subsection{Biochemical analysis of alginate beads}

Alginate beads were digested overnight at $56^{\circ} \mathrm{C}$ in papain buffer $(250 \mu \mathrm{g} / \mathrm{ml}$ papain in $50 \mathrm{mM}$ EDTA and $5 \mathrm{mM}$ L-cysteine). Glycosaminoglycan (GAG) amount in the digest was quantified using dimethylmethylene blue (DMB) assay (Farndale 1986). The metachromatic reaction of GAG with DMB was monitored with a spectrophotometer, and the ratio A530:A590 was used to determine the amount of GAG present, using 
chondroitin sulfate $\mathrm{C}$ (Sigma) as a standard. The amount of DNA in each papaindigested sample was determined using ethidium bromide with calf thymus DNA (Sigma) as a standard. High-performance liquid chromatography (HPLC) of amino acids (hydroxyproline, Hyp) and collagen cross-links (hydroxylysylpyridinoline, HP) was performed as described previously (Bank 1996; Beekman 1997). The quantities of cross-links were expressed as the number of residues per collagen molecule, assuming 300 Hyp residues per collagen triple helix. Three samples were taken of 7 alginate beads per experimental condition for biochemical analyses.

\subsection{Histochemical analysis of cartilage explants}

Formalin fixed cartilage constructs were embedded in paraffin. To prevent any negative influence of processing the constructs for histological analysis, separate $\underline{\text { cartilage constructs were placed in little porous polymer bags directly after harvesting }}$ prior to fixation in formalin and processing. However, a certain risk of damage during histological procedure is unavoidable. Therefore we embedded all the samples at the same time and did the sectioning in a random order to exclude bias. Sections $(6 \mu \mathrm{m})$ were cut using a standard microtome. Prior to the histological stainings, sections were deparaffinated in xylene and rehydrated through graded ethanol. For histological evaluation, serial sections were stained with Hematoxylin \& Eosin (H\&E). To evaluate integration, paraffin sections were stained with a thionine staining. For each sample we assessed the percentage of total interface length that had a matrixmatrix connection. A clear distinction could be made between parts with a matrix connection and parts of the cartilage touching each other but without a clearly connected matrix, which were scored as parts with a gap. Integration was determined at both integration sites within a paraffin section. Interface integration percentages 
were obtained by two blinded observers from measurements of three to four different sections from each sample, resulting in an average value for each interface.

\subsection{Statistical analysis}

For the alginate bead cultures and the integration study, four pooled cartilage donors were used. Statistical analysis was performed using GraphPad Prism 5.01 (GraphPad Software, San Diego, CA, USA) software. All data are presented as mean \pm standard deviation. Control groups and groups supplemented with PNPX, BAPN and/or IGF1 were compared with a ANOVA test followed by a post hoc Bonferroni test. 


\section{Results}

\subsection{Chondrocytes cultured in alginate}

After 42 days, DNA content, GAG deposition, collagen deposition and collagen crosslinking were determined in the alginate beads. In the control condition, DNA content was $0.49 \pm 0.03 \mu \mathrm{g}$ DNA per alginate bead, which was unaffected by the addition of BAPN, IGF1 and or PNPX during the first 21 days of culture (Figure 1).

$4.38 \pm 0.39 \mu \mathrm{g}$ GAG per bead was deposited in the control condition after 42 days of culture. As expected, this was significantly reduced in the condition with $0.25 \mathrm{mM}$ PNPX during the first 21 days. Compared to the control condition, GAG deposition was also less when a combination of PNPX and IGF1 was present, when PNPX and BAPN were present together, and when all three components were present in the culture medium. BAPN or IGF1 alone did not influence GAG deposition, rather their combination resulted in more GAG deposition (Figure 2).

Regarding the collagen deposition, $6.16 \pm 0.91 \mu \mathrm{g}$ was deposited in the control condition after 42 days. The presence of PNPX during the first 21 days resulted in less collagen deposition. Inhibition of collagen cross-link formation with BAPN on the other hand resulted in more collagen deposition, as expected. BAPN together with IGF1 increased the collagen deposition even more (Figure 3).

The presence of BAPN alone, BAPN with IGF1, BAPN with PNPX and all three factors during the first 21 days resulted in less cross-link formation, in line with the LOX inhibition by BAPN. Addition of IGF1 alone, PNPX alone or PNPX with IGF1 did not change the number of collagen cross-links from the control condition, which was $0.69 \pm 0.03 \mathrm{HP}$ per collagen triple helix (Figure 4).

\subsection{Cartilage integration}


In parallel to the alginate cultures, cartilage explants were cultured to investigate the integrative capacity in the presence of PNPX, BAPN and/or IGF1. As in the alginate cultures, soluble factors were only present during the first 21 days of culture followed by culture in control medium for an additional 21 days. Typical examples of integration after 42 days visualised with a thionine staining are shown in figure 5 . The average integration percentage in the control condition was less than $10 \%$. Only when PNPX, BAPN and IGF1 were combined during the first 21 days, integration of the two opposing cartilage explants improved to $41.3 \pm 31.4 \%(p<0.05)$. Although not reaching statistical significance, the presence BAPN and IGF1 alone or together also seems beneficial for integration. 


\section{Discussion}

Many factors have been demonstrated to be able to influence proteoglycan production, collagen production or both. However, it is not fully known what the optimal balance of collagen and proteoglycan production is and how they influence each other to form a functional network.

Our goal was to examine the effect of modulating collagen deposition, collagen cross-linking and GAG deposition simultaneously during cartilage matrix production and cartilage integrative repair, thereby creating a wider insight in the role of proteoglycans and collagen in cartilage repair process.

In the alginate cultures, inhibition of collagen cross-linking with BAPN in combination with promotion of matrix synthesis using IGF1 was most beneficial for matrix deposition. Additional PNPX was always detrimental for matrix deposition. For integration, the combination of BAPN, IGF1 and temporary prevention of proteoglycan formation with PNPX was most beneficial.

Inhibition of GAGs from binding to the proteoglycan core protein not only resulted in less GAG deposition in the alginate bead, but also in less collagen deposition. This is in concordance to our previous study were the presence of PNPX during a culture period of 35 days resulted in less proteoglycan and collagen deposition in the alginate bead. In addition, excretion of both matrix proteins into the culture medium was higher due to the absence of an intact matrix network (Bastiaansen-Jenniskens 2008b). Because of the absence of this network and the loss of interaction with other matrix components, combining PNPX with BAPN and/or IGF1 could not counteract the effects seen with PNPX alone. Earlier we found that the inhibition of collagen cross-linking in an alginate culture for 21 days and longer results in higher collagen deposition then when cross-links are formed. During this culture period, proteoglycan 
deposition was unaffected (Bastiaansen-Jenniskens 2008a). Even though cross-link inhibition by BAPN stimulates collagen synthesis, the retention of collagen is very low because of the absence of a proteoglycan network. Previously, we and others showed stimulating effects of IGF1 on collagen and proteoglycan deposition (Blunk 2002; Mauck 2003; De Mattei 2004; Jenniskens 2006). In the present setup, culture with IGF1 alone for 21 days did not significantly stimulate matrix deposition after 42 days, whereas IGF1 did previously already after 21 days (Jenniskens 2006). However, the combination IGF1 and BAPN increased GAG deposition which is probably attributable to the presence of IGF1 since BAPN does not affect the GAG deposition. Even though IGF1 might have also stimulated GAG and collagen deposition when combined with PNPX or with PNPX and BAPN, GAGs were prevented from incorporation into the matrix. A possible positive effect of IGF1 on collagen synthesis was counteracted because of low collagen retention in the alginate bead. In every condition where the cross-link inhibitor BAPN was present for the first 21 days, less collagen cross-links were present even though the culture continued for 21 days after removal of BAPN. This might be explained by the fact that after removal of BAPN, it takes three weeks for mature HP cross-links to be formed (Ahsan 2005). In addition, BAPN binds irreversibly to lysyl oxidase (LOX) (Tang 1983) and therefore extra time is also needed for LOX to be produced again. Based on our previous experiments, we expect that the number of collagen cross-links will be equal in all conditions when a longer culture period is used (BastiaansenJenniskens 2008a).

The combination of BAPN and IGF1 seems most beneficial for matrix synthesis when no matrix is formed yet, as in the alginate cultures. When aiming at the integration of two existing cartilage matrices, cross-link inhibition with BAPN and stimulation of 
matrix production with IGF1 is not sufficient to significantly improve integration. Transient inhibition of GAG incorporation in the matrix with PNPX in combination with BAPN and IGF1 did improve the integration. In these experiments, stimulation of matrix production alone with IGF1 had no effect. Transient inhibition of collagen cross-link formation could have resulted in more collagen synthesis, and in addition in better penetration of the newly formed collagen fibres in the opposing tissue that was partly depleted of GAGs following injury (Bos 2001). The latter is more likely since previous experiments with BAPN and cartilage explants did not result in more collagen synthesis (McGowan 2005). The study by McGowan et al is also in concordance with the theory of better integration after BAPN treatment. The addition of PNPX and the resulting absence of GAG attachment to the proteoglycan core protein might have similar effects as seen with inhibition of cross-link formation. Without an intact proteoglycan network at the edges of the cartilage explants, newly formed collagen fibres might penetrate better into the opposing cartilage tissue. Because of the absence of a proteoglycan network, the collagen network might also have a better integrity (Asanbaeva 2007). This explains why the presence of IGF1 with PNPX or BAPN did not improve integration since the newly formed collagen stimulated by IGF1 could not penetrate into the cartilage. PNPX together with BAPN but without IGF1 was also not sufficient because of the absence of increased collagen production.

$\underline{\text { When cartilage transplantations are performed, the surgeon removes the damaged }}$ cartilage first. Removing the damaged cartilage will create a fresh wound area, similar to our experimental condition. We hypothesize that the integrative capacity of such an area is different from a long lasting wound area and we are therefore convinced that our explant model is close to the application in this respect. 
The cartilage explants used for integration were cultured for 6 weeks in vitro. In an earlier study, we investigated the effect of improved integration on mechanical properties of the cartilage-cartilage interface (van de Breevaart Bravenboer 2004). There we observed a relation between the area of integration on histology and the mechanical properties of the interface. Since the previous study is performed after an $\underline{\text { in vivo culture period and our current study evaluates after in vitro culture, it is difficult }}$ to extrapolate these results. Earlier attempts in the lab have demonstrated us that after in vitro culture the bonding is not strong enough to measure reliably in our system. However, the improved histological integration in the present studies looks promising and suggests that when a construct is placed in vivo after modulation with our soluble factors (IGF1, PNPX and BAPN), integration might lead to functional cartilage. 


\section{Conclusions}

Based on these results, combining IGF1 and cross-link inhibition with BAPN seems to be most promising in promoting the formation of a new cartilage matrix. When a new matrix is produced, proteoglycans are important to retain collagen in the matrix. When two already formed cartilage matrices have to integrate, the temporary absence of a proteoglycan network and the temporary absence of collagen crosslinks might be more beneficial in combination with stimulation of collagen production by for example IGF1. Therefore, the choice of soluble factors to promote cartilage regeneration in OA depends on the type of therapy that will be used. In the case of cell therapy and the synthesis of a new matrix, stimulating both collagen and proteoglycan synthesis is important. The intra-articular administration of growth factors such as IGF1 to promote matrix production is the topic of ongoing research (Madry 2005) and could be a part of future OA therapy. When two cartilage matrices have to integrate, temporary inhibition of proteoglycan network formation and collagen cross-linking together with stimulating collagen production results in better integration. In the case of autologous cartilage transplantation, the transplant can be pre-treated with hyaluronidase or chondroitinase to remove the proteoglycans and be subjected to temporary collagen cross-link inhibition. To generate a tissue engineered cartilage construct, culture in the presence of IGF1 and BAPN might be a promising approach. However, care must be taken with BAPN since it is mutagenic. Other approaches to inhibit collagen cross-linking might be the topic of future research. 


\section{Figure legends}

Figure 1, DNA content of the alginate beads after 42 days of culture. PNPX (P), BAPN (B) and/or IGF1 (I) were present in the first 21 days of culture, followed by additional culture for 21 days in control medium. Data are shown as mean DNA content per bead \pm sd. $\mathrm{N}=3$.

Figure 2, GAG content of the alginate beads after 42 days of culture. PNPX (P), BAPN (B) and/or IGF1 (I) were present in the first 21 days of culture, followed by additional culture for 21 days in control medium. Data are shown as mean GAG content per bead \pm sd. $N=3$. * indicates a significant difference $(p<0.05)$ from the control condition without additives.

Figure 3, Collagen content of the alginate beads after 42 days of culture. PNPX (P), BAPN (B) and/or IGF1 (I) were present in the first 21 days of culture, followed by additional culture for 21 days in control medium. Data are shown as mean collagen content per bead \pm sd. $N=3 .{ }^{*}$ indicates a significant difference $(p<0.05)$ from the control condition without additives.

Figure 4, Number of HP cross-links per collagen triple helix after 42 days of culture. PNPX (P), BAPN (B) and/or IGF1 (I) were present in the first 21 days of culture, followed by additional culture for 21 days in control medium. Data are shown as mean HP per collagen triple helix $\pm \mathrm{sd} . \mathrm{N}=3 .{ }^{*}$ indicates a significant difference $(p<0.05)$ from the control condition without additives. 
Figure 5, Examples of integration visualised with a thionine staining. A) $20 \%$ integration in a control sample, B) $70 \%$ integration in a sample where PNPX, BAPN and IGF1 were present for the first 21 days. Arrows indicate integration, arrowheads indicate no integration. Magnification is $40 x$.

Figure 6, Percentage of integration of opposing cartilage tissue. PNPX (P), BAPN (B) and/or IGF1 (I) were present in the first 21 days of culture, followed by additional culture for 21 days in control medium. Data are shown as individual percentages with the mean percentage $\pm s d . N=8$. ${ }^{*}$ indicates a significant difference $(p<0.05)$ from the control condition without additives. 


\section{References}

Ahsan, T., F. Harwood, et al. (2005). Kinetics of collagen crosslinking in adult bovine articular cartilage. Osteoarthritis Cartilage 13(8): 709-15.

Aigner, T., W. Bertling, et al. (1993). Independent expression of fibril-forming collagens I, II, and III in chondrocytes of human osteoarthritic cartilage. J Clin Invest 91(3): 829-37.

Asanbaeva, A., K. Masuda, et al. (2007). Mechanisms of cartilage growth: modulation of balance between proteoglycan and collagen in vitro using chondroitinase ABC. Arthritis Rheum 56(1): 188-98.

Bank, R. A., E. J. Jansen, et al. (1996). Amino acid analysis by reverse-phase highperformance liquid chromatography: improved derivatization and detection conditions with 9-fluorenylmethyl chloroformate. Anal Biochem 240(2): 167-76.

Bastiaansen-Jenniskens, Y., W. Koevoet, et al. (2008b). Inhibition of glycosaminoglycan incorporation influences collagen network formation during cartilage matrix production. Biochem Biophys Res Commun In Press.

Bastiaansen-Jenniskens, Y. M., W. Koevoet, et al. (2008a). Contribution of collagen network features to functional properties of engineered cartilage. Osteoarthritis Cartilage 16(3): 359-66.

Beekman, B., N. Verzijl, et al. (1997). Synthesis of collagen by bovine chondrocytes cultured in alginate; posttranslational modifications and cell-matrix interaction. Exp Cell Res 237(1): 135-41.

Billinghurst, R. C., L. Dahlberg, et al. (1997). Enhanced cleavage of type II collagen by collagenases in osteoarthritic articular cartilage. J Clin Invest 99(7): 1534-45.

Blunk, T., A. L. Sieminski, et al. (2002). Differential effects of growth factors on tissueengineered cartilage. Tissue Eng 8(1): 73-84.

Bos, P. K., J. DeGroot, et al. (2002). Specific enzymatic treatment of bovine and human articular cartilage: implications for integrative cartilage repair. Arthritis Rheum 46(4): 97685. 
Bos, P. K., G. J. van Osch, et al. (2001). Growth factor expression in cartilage wound healing: temporal and spatial immunolocalization in a rabbit auricular cartilage wound model. Osteoarthritis Cartilage 9(4): 382-9.

De Mattei, M., A. Pellati, et al. (2004). Effects of physical stimulation with electromagnetic field and insulin growth factor-I treatment on proteoglycan synthesis of bovine articular cartilage. Osteoarthritis Cartilage 12(10): 793-800.

DiMicco, M. A., S. N. Waters, et al. (2002). Integrative articular cartilage repair: dependence on developmental stage and collagen metabolism. Osteoarthritis Cartilage 10(3): 218-25.

Farndale, R. W., D. J. Buttle, et al. (1986). Improved quantitation and discrimination of sulphated glycosaminoglycans by use of dimethylmethylene blue. Biochim Biophys Acta 883(2): 173-7.

Gooch, K. J., T. Blunk, et al. (2002). Bone morphogenetic proteins-2, -12, and -13 modulate in vitro development of engineered cartilage. Tissue Eng 8(4): 591-601.

Janssen, L. M., C. D. In der Maur, et al. (2006). Short-duration enzymatic treatment promotes integration of a cartilage graft in a defect. Ann Otol Rhinol Laryngol 115(6): 4618.

Jenniskens, Y. M., W. Koevoet, et al. (2006). Biochemical and functional modulation of the cartilage collagen network by IGF1, TGFbeta2 and FGF2. Osteoarthritis Cartilage 14(11): 1136-46.

Kaul, G., M. Cucchiarini, et al. (2006). Local stimulation of articular cartilage repair by transplantation of encapsulated chondrocytes overexpressing human fibroblast growth factor 2 (FGF-2) in vivo. J Gene Med 8(1): 100-11.

Lafeber, F. P., H. van Roy, et al. (1992). Human osteoarthritic cartilage is synthetically more active but in culture less vital than normal cartilage. J Rheumatol 19(1): 123-9.

Madry, H., G. Kaul, et al. (2005). Enhanced repair of articular cartilage defects in vivo by transplanted chondrocytes overexpressing insulin-like growth factor I (IGF-I). Gene Ther 12(15): 1171-9. 
Madry, H., R. Padera, et al. (2002). Gene transfer of a human insulin-like growth factor I cDNA enhances tissue engineering of cartilage. Hum Gene Ther 13(13): 1621-30.

Mauck, R. L., S. B. Nicoll, et al. (2003). Synergistic action of growth factors and dynamic loading for articular cartilage tissue engineering. Tissue Eng 9(4): 597-611.

McGowan, K. B. and R. L. Sah (2005). Treatment of cartilage with beta-aminopropionitrile accelerates subsequent collagen maturation and modulates integrative repair. $\mathrm{J}$ Orthop Res 23(3): 594-601.

Nelson, F., L. Dahlberg, et al. (1998). Evidence for altered synthesis of type II collagen in patients with osteoarthritis. J Clin Invest 102(12): 2115-25.

Tang, S. S., P. C. Trackman, et al. (1983). Reaction of aortic lysyl oxidase with betaaminopropionitrile. J Biol Chem 258(7): 4331-8.

van de Breevaart Bravenboer, J., C. D. In der Maur, et al. (2004). Improved cartilage integration and interfacial strength after enzymatic treatment in a cartilage transplantation model. Arthritis Res Ther 6(5): R469-76.

Veilleux, N. and M. Spector (2005). Effects of FGF-2 and IGF-1 on adult canine articular chondrocytes in type II collagen-glycosaminoglycan scaffolds in vitro. Osteoarthritis Cartilage 13(4): 278-86.

Verzijl, N., J. DeGroot, et al. (2000). Effect of collagen turnover on the accumulation of advanced glycation end products. J Biol Chem 275(50): 39027-31.

Wong, M., M. Siegrist, et al. (2002). Collagen fibrillogenesis by chondrocytes in alginate. Tissue Eng 8(6): 979-87. 
Figure 1

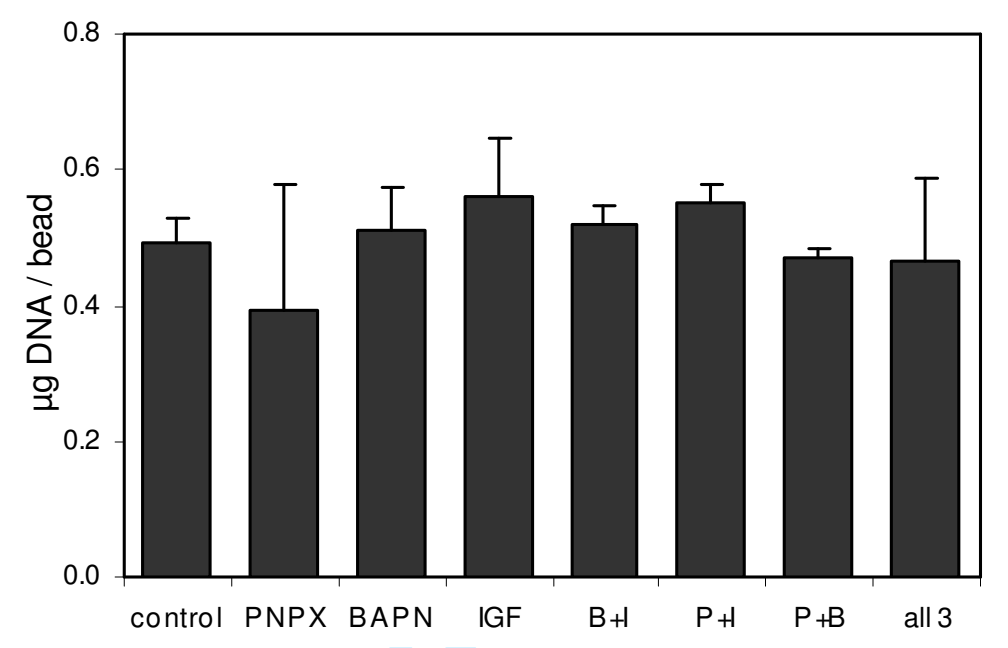

2

4

5

6

7

8

9

10

11

12

13

14

15

16

17

18

19

20

21

22

23

24

25

26

27

28

29

30

31

32

33

34

35

36

37

38

39

40

41

42

43

44

45

46

47

48

49

50

51

52

53

54

55

56

57

58

59

60 
Figure 2

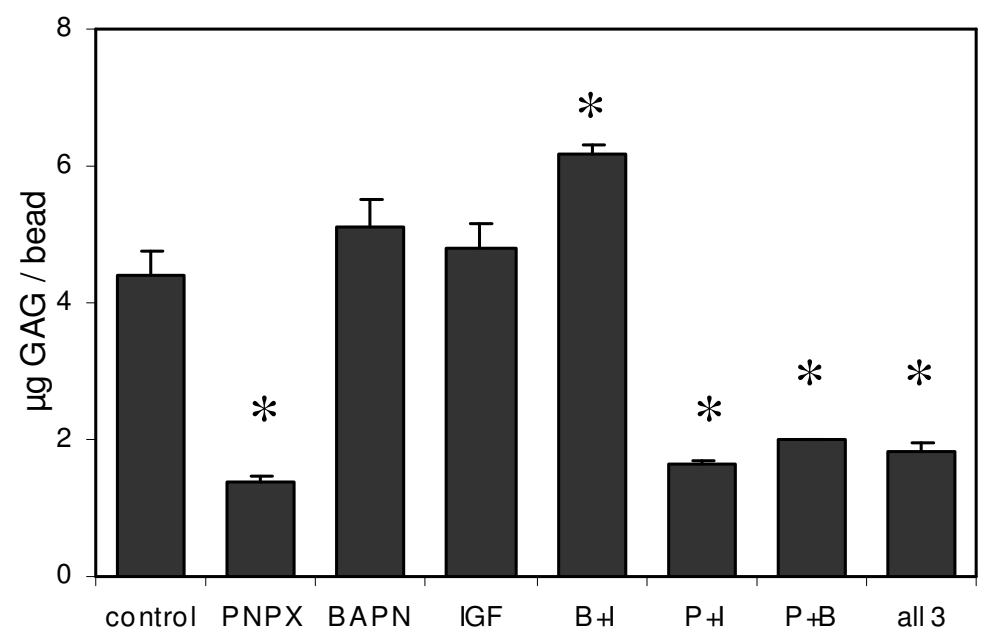

21

22

23

24

25

26

27

28

29

30

31

32

33

34

35

36

37

38

39

40

41

42

43

44

45

46

47

48

49

50

51

52

53

54

55

56

57

58

59

60

http://mc.manuscriptcentral.com/term 
Figure 3

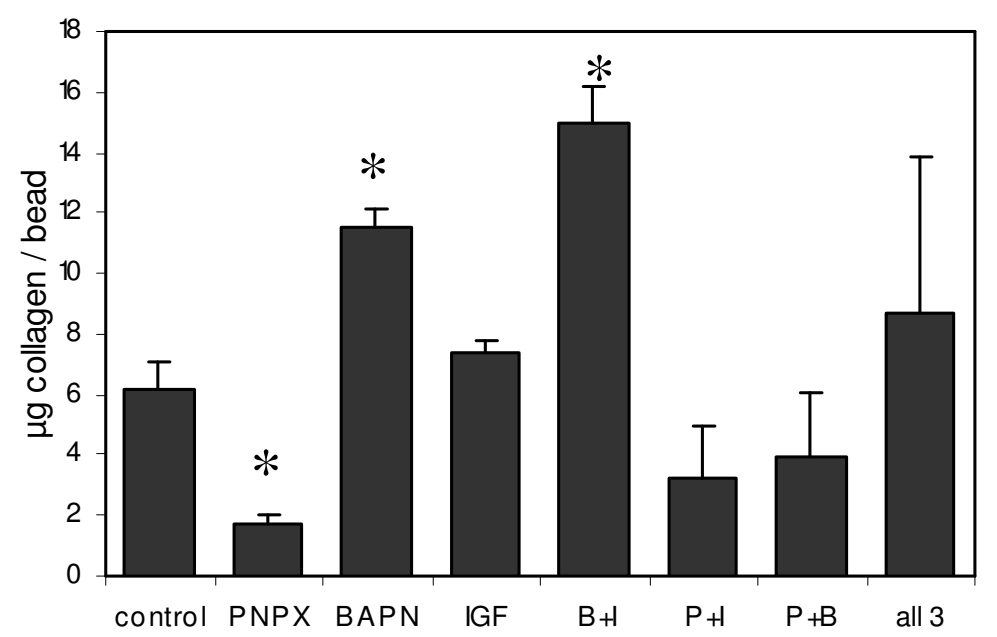

21

22

23

24

25

26

27

28

29

30

31

32

33

34

35

36

37

38

39

40

41

42

43

44

45

46

47

48

49

50

51

52

53

54

55

56

57

58

59

60

http://mc.manuscriptcentral.com/term 
Figure 4

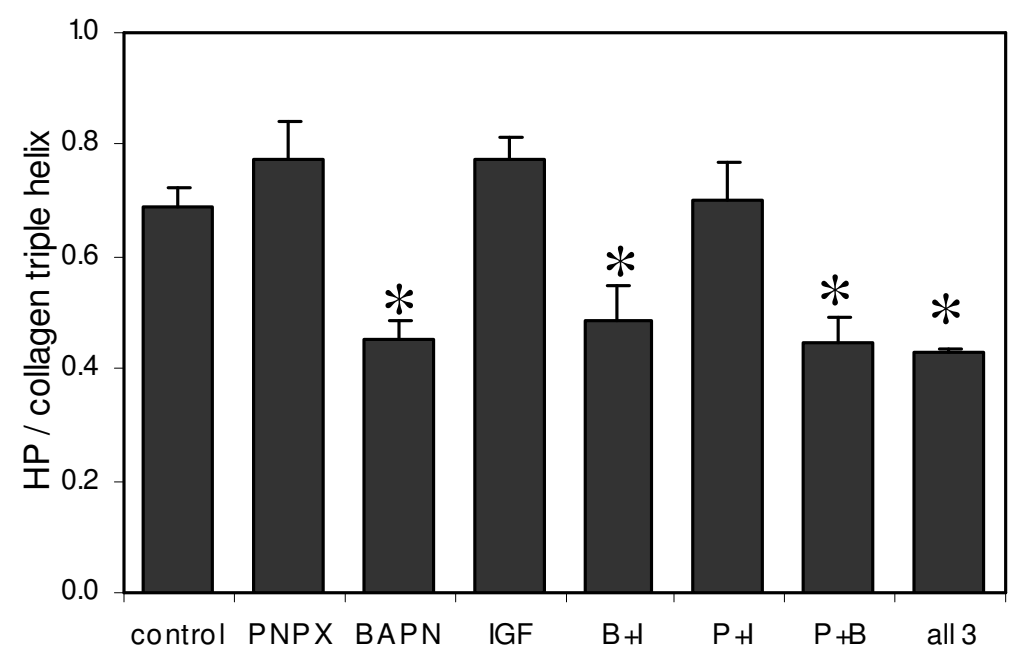

2

4

5

6

7

8

9

10

11

12

13

14

15

16

17

18

19

20

21

22

23

24

25

26

27

28

29

30

31

32

33

34

35

36

37

38

39

40

41

42

43

44

45

46

47

48

49

50

51

52

53

54

55

56

57

58

59

60

http://mc.manuscriptcentral.com/term 
Figure 5

A
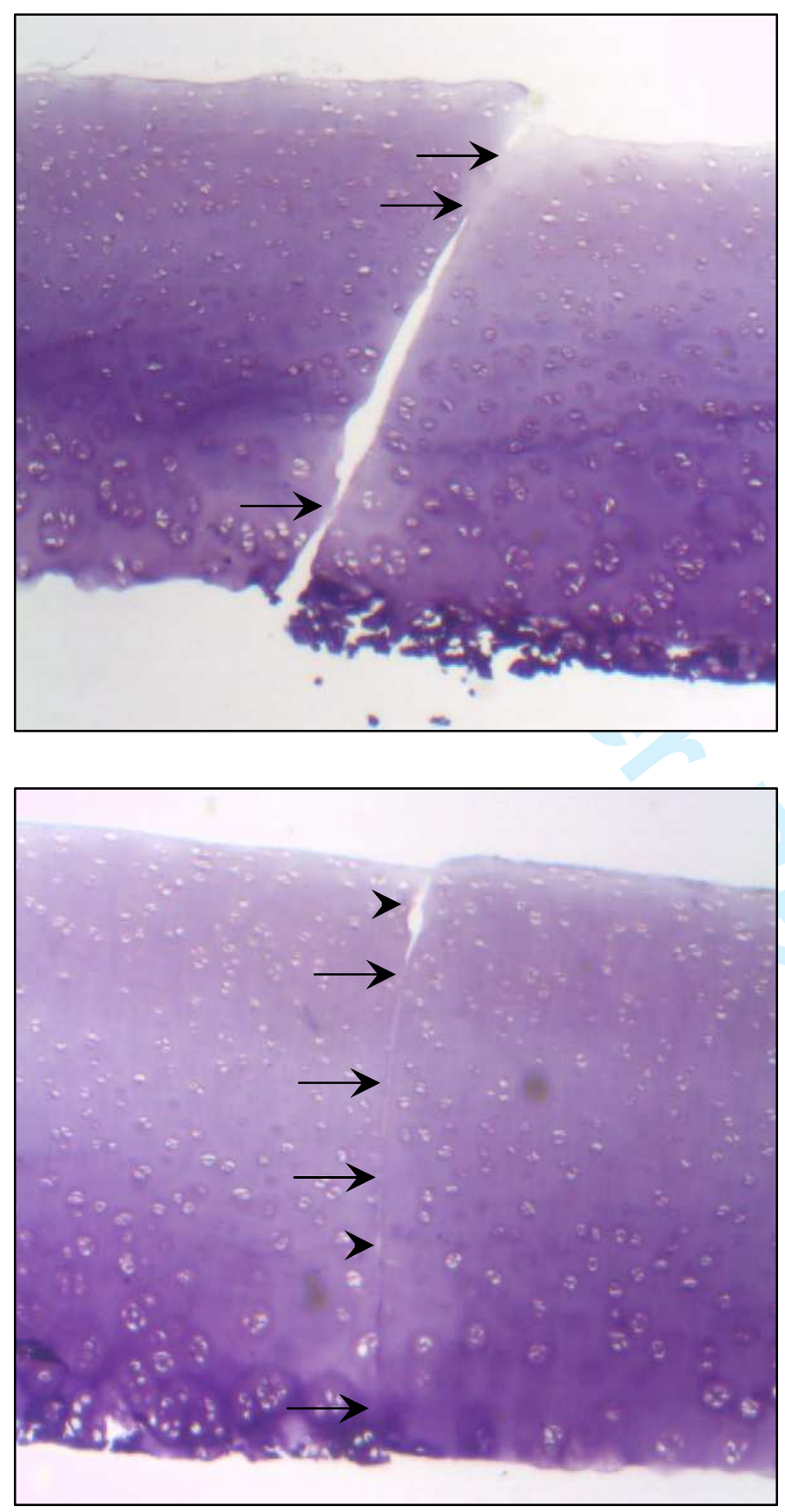

http://mc.manuscriptcentral.com/term 
Figure 6

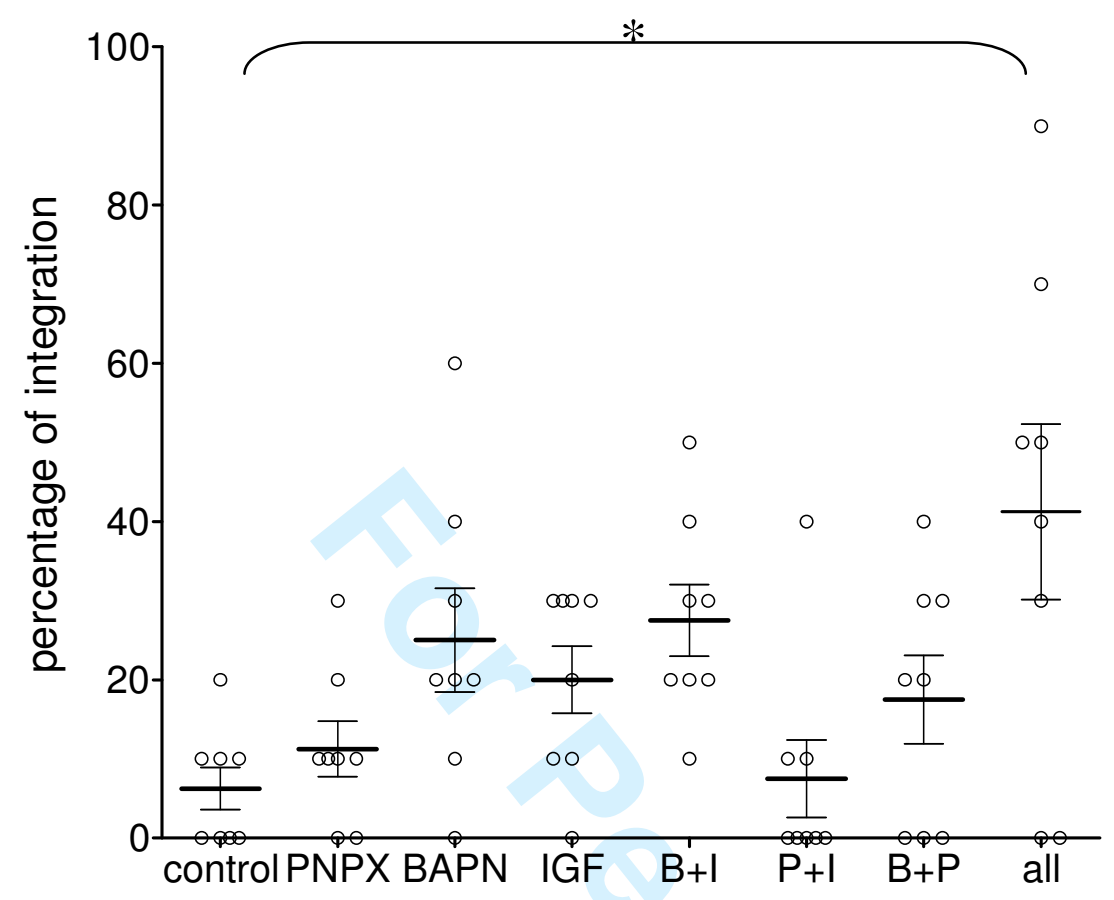

29

30

31

32

33

34

35

36

37

38

39

40

41

42

43

44

45

46

47

48

49

50

51

52

53

54

55

56

57

58

59

60

http://mc.manuscriptcentral.com/term 